\title{
A mathematical model of cellular swelling in Neuromyelitis Optica
}

Simão Laranjeira ${ }^{1}$, Mkael Symmonds $^{2}$, Jacqueline Palace ${ }^{2}$, Stephen J. Payne ${ }^{1}$, Piotr Orlowski ${ }^{1}$

${ }^{1}$ Department of Engineering Science, University of Oxford, UK

${ }^{2}$ Department of Clinical Neurology, University of Oxford, UK

Correspondence:

Piotr Orlowski

Institute of Biomedical Engineering, University of Oxford

Old Road Campus Research Building

OX3 7DQ, Oxford

United Kingdom

Phone: +441865271025

E-mail: piotr.orlowski@eng.ox.ac.uk

Fax: +441865617701 


\title{
Abstract and key terms
}

\begin{abstract}
Neuromyelitis Optica (NMO) is a severe neuro-inflammatory disease of the central nervous system characterized by predominant damage to the optic nerve and of the spinal cord. The pathogenic antibody found in the majority of patients targets the AQP4 channels on astrocytic endfeet and causes the cells to swell. Although, the pathophysiology of the disease is broadly known, there are no specific targeted treatments for this process clinically available nor accurate prognostic markers both during attacks and for predicting long term neuronal damage. This lack is, in part, due to the rarity of the disease and its relatively recent pathogenic clarity. Hence, the ability to mathematically model the progress of the condition to test prospective therapies in silico would be a step forward. This paper combines state of the art models of cellular metabolism and cytotoxic oedema in neurons and astrocytes and augments it with a detailed characterization of water transport across the cellular membrane. In particular, we capture the process of perforation of the cell through the human complement cascade and resulting water and ionic fluxes. Simulating NMO by injecting its antibody and human complement into the extracellular space showed a $25 \%$ increase of the astrocytic volume after 12 hours from onset. Most of the volume change occurred during the first 30 minutes of simulation with a peak volume change of $38 \%$. The model was further adapted to simulate the therapeutic potential of C59. It was found that there is a threshold of CD59 concentration that can prevent the swelling of astrocytes. Since the astrocyte volume changes mostly during the first hour, further experimental work should focus on this time scale to provide data for further model refinement and validation.
\end{abstract}

\section{Key Terms}

Neuromyelitis Optica, CD59, Cytotoxic oedema, Complement induced lysis, AQP4 kinetics. 


\section{Introduction}

Neuromyelitis optica (NMO) is a neuro-inflammatory central nervous system disease characterized by predominant damage to the optic nerve and of the spinal cord. Although rare, with a worldwide incidence of approximately 1 per 100,000, NMO is severe, with a 5year mortality rate of $32 \%$ in patients with a relapsing disease.

The IgG1 antibody targeting aquaporin-4 (AQP4) water channels located at the endfeet of astrocytes (Papadopoulos et al., 2014). The antibody is present in about $60 \%$ of NMO patients and is thought to be pathogenic causing the primary astrocytopathy (Fujihara et al., 2012). A mouse model has shown that both AQP4-Ab and human complement in the extracellular space are needed to initiate the pathophysiological process during which astrocytes swell within twelve hours (Saadoun et al., 2010). The swelling has been shown to be due to complement induced membrane lytic holes which facilitate metabolite transport over the membrane and lead to cell growth (Saadoun et al., 2010). In turn, the swelling triggers an inflammatory response marked by granulocyte and macrophage infiltration leading to secondary oligodendrocyte damage, demyelination and neuronal death (Papadopoulos et al., 2014).

There is currently no cure for NMO. Treatment focuses on either the management of the acute phase of the condition or the prevention of relapses using generic immunosuppressive or immunomodulatory treatments (Kitley and Palace, 2016). In the former case, the most effective method, out of those trailed, consists of complementary intravenous injection of methylprednisolone (IVMP) and plasma exchange. The most comprehensive study on the therapeutic effects of plasma exchange is that by Kleiter et al. (2016). The study consists of the analysis of 59 CNS demyelination patients, where 10 had NMO, who were treated with plasma exchange for acute severe attacks from 1984 to 2000 . It found that $44.1 \%$ of patients experienced moderate to marked functional improvement.

Current developments focus on inhibiting the breakdown and binding of a range of proteins in the complement cascade. For example, eculizumab, binds to C5 and inhibits its cleavage thus preventing further mechanisms leading to membrane lysis. In a pilot study performed by (Pittock et al., 2013), 14 relapsing NMO patients were treated with eculizumab for 12 months. During that period the average number of relapses fell from 3 to zero. However, this non-controlled data requires larger randomized controlled trials to assess true efficacy, which has large resource and cost implications and a greater risk of predictable and unpredictable adverse effects. For example the risk of infection with encapsulated bacteria such as meningococcal infection which is potentially fatal even after vaccination (Lewis and Ram, 2014).

A promising strategy for complement inhibition is the upregulation of the astrocytic complement inhibitor CD59, which inhibits C5b-9 formation as described by (Davies and Lachmann, 1993). CD59 is naturally present in astrocytes. However, its concentration has been reported to be smaller in proximity of the endfeet where the NMO antibody acts. For this reason it is expected that CD59 will have fewer side effects compared to the clinically used therapies above(Zipfel and Skerka, 2009).

To evaluate the potential of CD59 there is a need to optimize its dosage, and their administration timing. Testing combined effects of novel drugs at different doses at different stages in disease evolution is impossible in patient cohorts, due to both disease rarity and 
the ethical contraventions in withholding conventional immunosuppression rather than placebo, which is likely to reduce the power to detect a further treatment effect. Furthermore, testing these effects in animal studies or in vitro models is costly, impractical and limited in scope. In silico models offer the promise of being able to search for optimal therapeutic regimens and the timing of administration of drugs in simulated environments that can capture the core features of the pathogenic process in disease. However, these methods have been considerably underutilized for studying inflammatory disease.

Hence, we introduce a mathematical model of the swelling of astrocytes during NMO and its modulation by CD59. To capture the pathological cell volume evolution, the model extends a state of the art model of metabolism in astrocytes and neurons with a detailed representation of water transport across the cell membrane due to osmotic and hydrostatic pressure. In particular, in addition to representing transport through AQP4 channels and water permeable ionic channels, we model the perforation of the cellular membrane due to NMO specific complement lysis. CD59 acts as a modulator of the complement cascade.

We demonstrate the fidelity of our computational model of the neuroinflammatory environment by demonstrating that the simulation predicts that the primary pathogenic mechanism in NMO is complement induced lysis rather than direct inactivation of AQP4 channels, a fact now experimentally confirmed (Saadoun et al., 2010) but originally contentious when the disease was first described. Moreover, we simulate the degree of astrocyte swelling due to complement induced lysis, and map out the time course of this process, and then simulate the impact of a putative therapeutic strategy - CD59 injection on the cellular swelling.

\section{Methods}

\subsection{Astrocyte swelling model}

Assuming that the intracellular space is composed mostly of water (Blaivas, 1998) and that water is an incompressible fluid, the rate of change of the cell volume $V$ is assumed to be equal to the net water flux $Q$ across the cell membrane:

$$
\frac{\partial V}{\partial t}=Q
$$

According to the Starling equation (Jiang and Sun, 2013) the water flux across the cell membrane is a function of the osmotic $(\Delta \pi)$ and hydrostatic $(\Delta H)$ pressure gradients between the inside and outside of the cell:

$$
Q=\frac{A M}{R T} k[\Delta H-\Delta \pi]
$$

where $A$ is the cell's surface area, $M$ is the molecular weight of water, $R$ is the gas constant, $T$ is temperature and $k$ is the permeability of the cell to water which depends on the density and type of channels through, which water can flow. In the cellular 
context considered the majority of water flow is driven by $\Delta \pi$. Although, hydrostatic pressure gradient is negligible, $\Delta H$ is kept in Eq. 2 to facilitate the application of the model in other contexts, e.g. hydrocephalus (Desai et al., 2016), where hydrostatic pressure driven transport can be significant.

According to a recent review by Nagelhus and Ottersen (2013) there are two known types of channels transporting water: water specific aquaporin channels and ionic channels permeable to water. Further water flows through non-specific pathways. Hence, the net membrane flux can be expressed as:

$$
Q=Q_{A Q P 4}+Q_{i c}+Q_{n s}
$$

where the indices $A Q P 4$, ic and $n s$ stand for the AQP4, ionic channel and non-specific channels respectively.

\subsubsection{Flux through AQP4 channels}

AQP4 is the aquaporin subtype expressed in astrocytes. AQP4 can be oriented towards the vasculature at the astrocytic endfeet and towards the extracellular space (ECS). In the model created here these two orientations were considered by defining the flux through AQP4 channels as:

$$
Q_{A Q P 4}=\frac{A M}{R T}\left[\left(\gamma k_{A Q P 4}\right)\left[\Delta H_{c g}-\Delta \pi_{c g}\right]-\left((1-\gamma) k_{A Q P 4}\right)\left[\Delta H_{g e}-\Delta \pi_{g e}\right]\right] \text {, }
$$

where the indices ?, $e$ and $g$ stand for the capillary, the ECS and the astrocyte respectively, $\gamma$ stands for the proportion of AQP4 channels at the perivascular interface and $k_{A Q P 4}$ is the permeability of AQP4 channels. The parameter $\gamma$ was assumed to be equal to 0.8 based on the work by Nagelhus and Ottersen (2013) that states that the majority of AQP4s are oriented towards the perivascular space. The value of $k_{A Q P 4}$ was defined from the work by Solenov et al. (2004), which measured astrocytic membrane permeability when astrocytes are grown both with AQP4 and without. It was found that there is a 7-fold reduction of membrane permeability when AQP4 is eliminated. Finally, hydrostatic pressures in capillaries and the ECS is defined based on the work by Kimelberg (2004), stating average pressures of $15 \mathrm{mmHg}$ and $7.5 \mathrm{mmHg}$ respectively.

\subsubsection{Water flux through ionic channels}

According to Nagelhus and Ottersen (2013) the two ionic channels currently known to be permeable to water in astrocytes are the glutamate transporter (GTR1) and the sodium-potassium-chloride co-transporter (NKCC1).

Several studies have considered the kinetics of the NKCC1 channels and their involvement in the clearance of potassium after neuronal stimulation (Dronne et al., 2006; Jin et al., 2013; Østby et al., 2009). However, only the Dronne et al. (2006) model considered both the glutamate transporter (GLT1) and the NKCC1 channel during swelling caused by cytotoxic oedema. Hence, their model is adopted here. Based on their work, ionic currents through the channels are modelled with the Goldman equation. Accounting for the stoichiometry of the transport, the Nernst potentials are defined as 


$$
\begin{gathered}
E_{G T R 1}=\frac{R T}{F} \ln \left(\frac{\left[G L U^{-}\right]_{e}}{\left[G L U^{-}\right]_{i}} \frac{\left[K^{+}\right]_{i}}{\left[K^{+}\right]_{e}}\left(\frac{\left[N a^{+}\right]_{e}}{\left[\mathrm{Na}^{+}\right]_{i}}\right)^{3}\right), \\
E_{N K C C}=\frac{R T}{F} \ln \left(\frac{\left[\mathrm{Na}^{+}\right]_{e}}{\left[\mathrm{Na}^{+}\right]_{i}} \frac{\left[K^{+}\right]_{e}}{\left[\mathrm{~K}^{+}\right]_{i}}\left(\frac{[\mathrm{Cl}]_{e}}{\left[C l^{-}\right]_{i}}\right)^{2}\right),
\end{gathered}
$$

where $\left[\mathrm{GLU}^{-}\right],\left[\mathrm{K}^{+}\right],\left[\mathrm{Na}^{+}\right]$and $\left[\mathrm{Cl}^{-}\right]$are the concentrations of glutamate and potassium, sodium and chloride ions respectively in the extra and intra cellular space as indicated by indices $e$ and $i$. The ionic currents through the cell membrane are then defined as:

$$
\begin{gathered}
i_{G T R 1}=-S_{g} k_{G T R}\left(v_{g}-E_{G T R}\right) \\
i_{N K C C}=-S_{g} k_{N K C C} E_{N K C C}
\end{gathered}
$$

where $S_{g}$ is the surface area of the astrocyte, $v_{g}$ is the astrocyte membrane potential and $k_{G T R}$ and $k_{N K C C}$ are permeabilities of the two channels. According to Nagelhus and Ottersen (2013) the transport of one mole of each ion through their respective channels is accompanied with transport of one mole of water. Hence, water flux $Q_{i c}$ is proportional to these ionic currents as

$$
Q_{i c}=M \frac{i_{G T R}+i_{N K C C}}{V F}
$$

where $V$ is the total volume considered in Cloutier et al. (2009) model and $F$ is the faraday constant.

\subsubsection{Water flux through non-specific pathways}

The flux through non-specific pathways has been assumed to be proportional to the hydrostatic and osmotic pressure gradients across the membrane with the same permeability $k_{0}$ across the perivascular and ECS interfaces as

$$
Q_{n s}=k_{0} \frac{A M}{R T}\left[\left[\Delta H_{c g}-\Delta \pi_{c g}\right]-\left[\Delta H_{g e}-\Delta \pi_{g e}\right]\right] .
$$

\subsection{Extension of the model with metabolism}

Osmotic pressure in Eq. 2, Eq. 4 and Eq. 10 is a function of the difference of the concentration of cell metabolites. Furthermore, the current through water permeable ionic channels (Eq. 4 and Eq. 5) depends on $\left[\mathrm{GLU}^{-}\right],\left[\mathrm{Cl}^{-}\right],\left[\mathrm{Na}^{+}\right]$and $\left[\mathrm{K}^{+}\right]$. Hence, there is a need to capture the regulation of the concentration of these molecules to simulate glial swelling and to represent their change in both the intracellular space and the ECS.

The concentration of ions in the ECS is affected by transport across neurons and astrocytes. Hence, there is a need to further add a neuron compartment to the model. Transport of water occurs at both the interface of the cell and the ECS and of blood vessels further increasing the need to include a capillary compartment in the model.

An existing model by Orlowski et al. (2013) based on the previous work by Cloutier et al. (2009) has been used to simulate neural and glial swelling during stroke. The Cloutier et 
al. (2009) model is composed of four compartments: the astrocyte, the neuron, the capillary and the ECS. Glycolysis is represented in both cells through a 6-step chemical reactions chain transforming glucose into pyruvate. Pyruvate, in turn is used to recharge ATP in the cells' mitochondria to fuel the sodium potassium pump and for cell general maintenance. The capillary compartment supplies the tissue with oxygen and glucose and evacuates $\mathrm{CO}_{2}$ and lactate.

The Cloutier et al. (2009) was developed to simulate brain metabolic reactions in the presence of neuronal stimulation. The current model considers conditions of metabolic stress that the Cloutier model does not account for. Therefore, the model was adapted in order to be able to simulate ATP consumption in the presence of ischaemic stroke. This analysis is fully described in the supplementary material.

Additionally, the Cloutier et al. model was expanded with relevant modifications made by Orlowski et al. (2013) included adding $\mathrm{Na}^{+}, \mathrm{Ca}^{2+}, \mathrm{K}^{+}$and $\mathrm{Cl}^{-}$transport across the membrane and water transport due to osmotic pressure. However, the water flow model did not include kinetics of water permeable channels. Hence, in this model we use the Cloutier et al. (2009) model as a basis, expand it with the valid ion model from Orlowski et al. (2013) and further expand it with the water transport model from the previous section. Furthermore, to capture glutamate transport the model of (Dronne et al., 2006) was used. The overall model is represented in Figure 1.

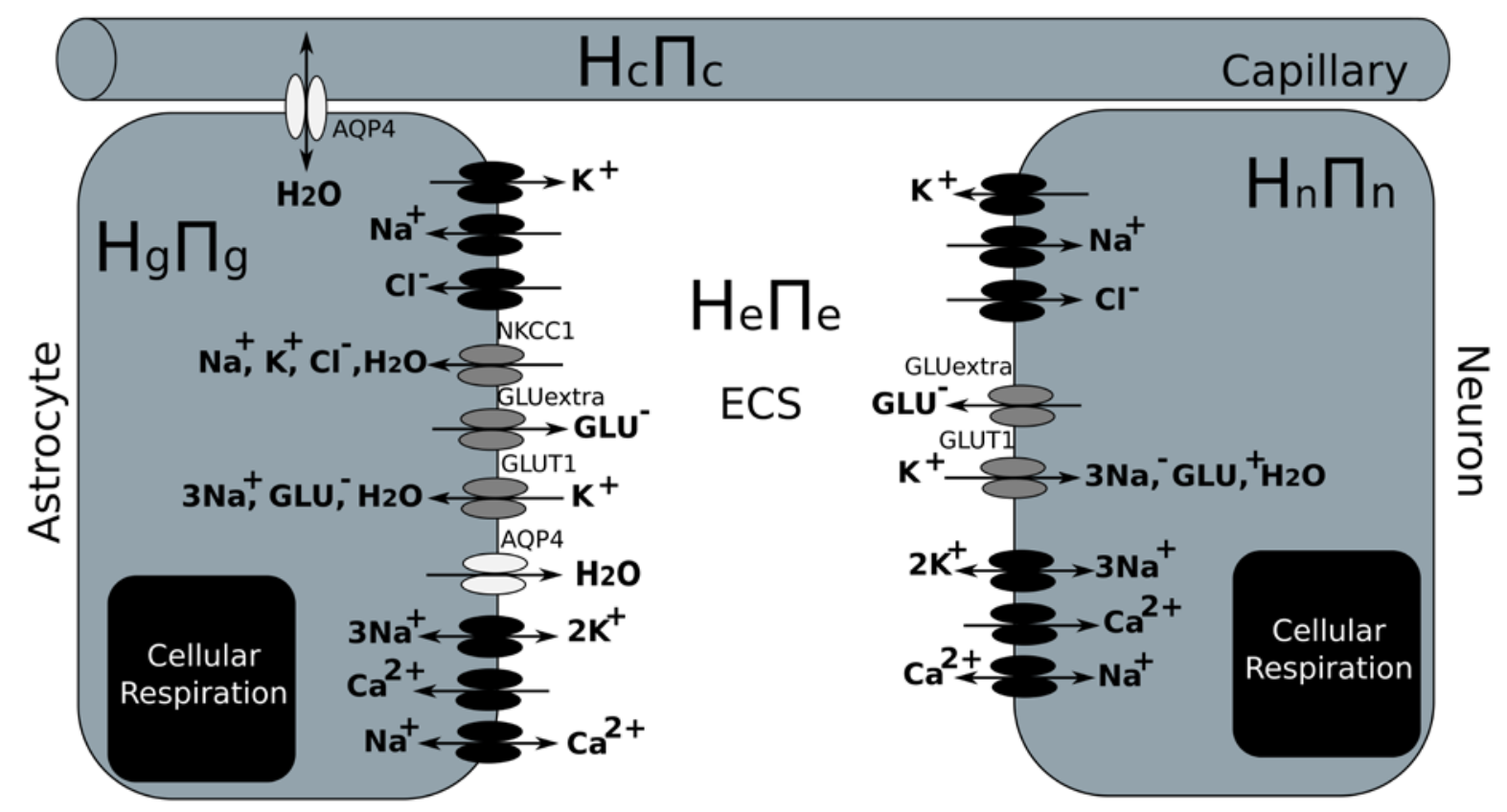

Figure 1: Diagram summarizing the astrocyte swelling model, which consists in the combination of three models: 1 . The Orlowski et al. (2013) model of cytotoxic oedema (green) 2. Ionic channels responsible for water movement (blue) was based on the work by Dronne et al. (2006) and 3. Water kinetics through AQP4 channels (red) based on the work by Kimelberg (2004).

\subsubsection{Neuron integration}

The new model includes both an astrocyte and a neuron compartment. All equations defined for water transport and production in Section 2.1 apply to the neuron compartment 
with two exceptions: according to Nagelhus and Ottersen (2013) there is no AQP4 interface between the capillary and the neuron; and there are no NKCC1 channels in the neuron membrane.

\subsubsection{Glutamate channel}

In the Cloutier et al. (2009) model, glutamate kinetics are designed to capture the neurotransmitter release during an action potential and were replaced to be suitable for the swelling cell context. The formulation by Dronne et al. (2006) was used as they model cytotoxic oedema in stroke. In this model, both neurons and astrocytes uptake glutamate through the $i_{G T R 1}$ channels. The current $i_{G L U}$ through this channel is activated following the cell's membrane depolarization as defined by:

$$
i_{G L U}=S_{x} k_{G L U_{x}}\left(v_{x}-E_{G L U}\right)
$$

where the Nernst potential $E_{G L U}$ is defined as

$$
E_{G L U}=\frac{R T}{F} \ln \left(\frac{\left[G L U^{-}\right]_{e}}{\left[G L U^{-}\right]_{i}}\right)
$$

After adding the extra currents into the ionic model by Orlowski et al. (2013) (Figure 1 blue and red channels) the permeability of all ionic channels was refitted to ensure ion concentrations are balanced at initialization. As above, the simplex method was used to perform the fit. The full refitted model is provided in the supplementary material.

\subsubsection{Osmotic pressure}

Following Orlowski et al. (2013), the intracellular osmotic pressure is defined as being proportional to the sum of the ionic concentrations in each compartment as:

$$
\pi=\delta R T\left(\left[\mathrm{Na}^{+}\right]+\left[\mathrm{K}^{+}\right]+\left[\mathrm{GLU}^{-}\right]+\left[\mathrm{Ca}^{2+}\right]+\left[\mathrm{HCO}_{3}^{-}\right]+\left[\mathrm{A}^{-}\right]\right)
$$

where $\delta$ is the van't Hoff factor, $\left[\mathrm{K}^{+}\right],\left[\mathrm{Na}^{+}\right],\left[\mathrm{HCO}_{3}^{-}\right],\left[\mathrm{Ca}^{2+}\right]$ and $\left[\mathrm{GLU}^{-}\right]$are the potassium, sodium, carbonate, calcium and glutamate ionic concentrations respectively. Additionally, an assumption is made that there are impermeable anions $\left[A^{-}\right]$inside the cells. The initial concentrations of these anions, following the work by Orlowski et al. (2013) and Østby et al. (2009), are calculated to ensure that the net pressure across the cells membranes is zero at the steady initial state. This procedure is explained in the supplementary material.

\subsection{Membrane lysis model}

The model of membrane lysis by AQP4-Ab and complement captures three key mechanisms: 1) the binding of AQP4-Ab antibodies to AQP4 antigens, 2) the complement protein cascade leading to the production of the Membrane Attack Complex 
(MAC) forming holes in the membrane; and 3) the ionic and water currents through the forming membrane holes. The three-step mechanism is summarized in Figure 2.

\begin{tabular}{|cccccc|}
\hline Astrocyte & AQP4-Ab & AQP4 & Complement & MAC & Hole \\
\hline & $\lambda$ & 0 & & $\square$ & $\square \square$
\end{tabular}

Human AQP4-Ab and human complement are injected into the ECS.

1) The antibody attaches to AQP4 and then the complement protein cascade is initiated

The complement system starts

(2) producing MAC which perforates the glial membrane

In the end MAC forms holes on the

3 membrane allowing ions to permeate it causing astrocytes swell

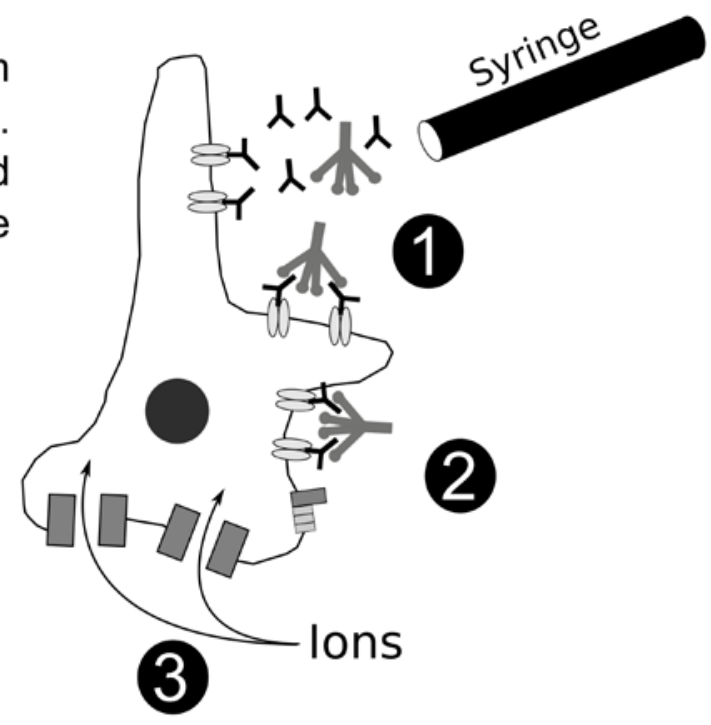

Figure 2. Summary of the three-step model of hole formation during NMO.

\subsubsection{Antigen antibody binding}

The production of the AQP4-Ab and AQP4 complex $\left(C_{I g G, A Q P 4}\right)$ was modelled using the generic model of antibody antigen binding developed by Kumagai and Tsumoto (2010):

$$
A Q P 4_{I g G}+A Q P 4_{\substack{\rightarrow \\ k_{b}}}^{\stackrel{k_{d}}{\leftarrow}} C_{I g G, A Q P 4}
$$

where $\boldsymbol{k}_{\boldsymbol{b}}$ and $\boldsymbol{k}_{\boldsymbol{d}}$ binding and detachment rates were set to the middle value of the range reported by Kumagai and Tsumoto (2010): $10^{2}-10^{3} \mathrm{mM}^{-1} \mathrm{~s}^{-1} \boldsymbol{k}_{\boldsymbol{b}}$ and $10^{-4}-10^{-3} \mathrm{~s}^{-1}$ for $\boldsymbol{k}_{\boldsymbol{d}}$. The concentration of AQP4 is set to be equal to

$$
[A Q P 4]_{g}=\frac{n_{A Q P 4}}{N_{A} V_{m}}
$$

where $n_{A Q P 4}$ is the number of AQP4 channels found in the membrane of the astrocyte, $N_{A}$ is the Avogadro number and $V_{m}$ is the volume of the astrocyte membrane. $V_{m}$ was calculated based on measurements of the average astrocyte surface area in Dronne et al. (2006) $\left(2.09241 \times 10^{-7} \mathrm{dm}^{2}\right)$ and measurements of membrane thickness in Curtis and Barnes (1989) (8 nm). Hence, $[A Q P 4]_{g}$ was set to $3.97 \times 10^{-3} \mathrm{mM}$. 


\subsubsection{MAC production}

The process responsible for the perforation of the membrane of astrocytes during $\mathrm{NMO}$ is the inflammatory complement system triggered by the interaction of AQP4-Ab and AQP4 (Saadoun et al., 2010). The holes are formed through the production of a membrane piercing protein: the Membrane Attack Complex (MAC).

The complement system, as described by Kumar et al. (2012), comprises three pathways, each of which have different triggers. The classical pathway is activated by the presence of antigen-antibody complexes. The alternative pathway is also activated by the antigen-antibody complexes; however, it diverges from the classical pathway due to the cleavage of $\mathrm{C} 3$ protein to form $\left.\mathrm{C} 3 \mathrm{CH}_{2} \mathrm{O}\right)$ resulting in a different protein cascade. Finally, there is the lectin pathway, which is activated by the detection of surface molecules by specialized proteins called serine proteases.

Here, only the classical pathway is considered, as it is the only one known to be activated during NMO (Papadopoulos et al., 2014). The classical pathway consists of the breakdown and binding of a cascade of proteins in the following steps (Kumar et al., 2012): 1) the protein C1 binds to the antibody-antigen complex which in turn activates the cleavage of $\mathrm{C} 4$ and $\mathrm{C} 2$ into $\mathrm{C} 4 \mathrm{~b}$ and $\mathrm{C} 4 \mathrm{a}$, and $\mathrm{C} 2 \mathrm{a}$ and $\mathrm{C} 2 \mathrm{~b}$ respectively; 2) C4b and $\mathrm{C} 2 \mathrm{a}$ form a complex and bind to the astrocytic surface, which initiates the breakdown of C3, C3a being released into the ECS and acting as an inflammatory chemoattractant while the protein $\mathrm{C} 3 \mathrm{~b}$ binds to the $\mathrm{C} 4 \mathrm{~b} 2 \mathrm{a}$ complex; 3 ) the binding leads to the cleavage of $\mathrm{C} 5, \mathrm{C} 5 \mathrm{a}$ is released into the ECS also behaving as chemoattractant and the protein $\mathrm{C} 5 \mathrm{~b}$ instead binds to the astrocytic membrane enrolling the proteins C6, C7 and C8 resulting in the formation of C5bC6C7C8 complex (C5b-8); 4) the final step consists of the activation $\mathrm{C} 9$ proteins these binding to the $\mathrm{C} 5 \mathrm{~b}-8$ complex or MAC and perforate the astrocytic membrane. The steps of MAC formation are summarized in Figure 3.

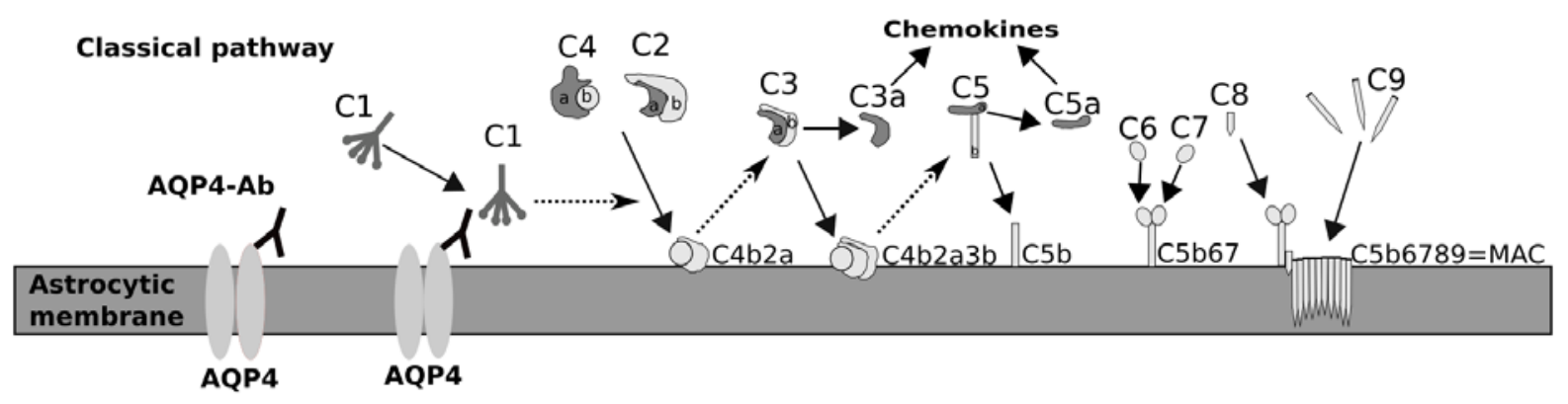

Figure 3: Breakdown of the classical complement pathway protein cascade leading to the formation of the membrane attack complex.

In the literature there are three models that capture the protein cascade of the complement system, Hirayama et al. (1996), Korotaevskiy et al. (2009) and Liu et al. (2011). In Korotaevskiy et al. (2009) and Liu et al. (2011) it was found that the model in Hirayama et al. (1996) cannot reproduce experimental data. Alternatively, the work of Liu et al. (2011), although it has validated its model with experimental data, does not model the full pathway from complement activation to the formation of MAC. Hence the model of Korotaevskiy et al. (2009) was chosen.

Two adaptations were made to the model of Korotaevskiy et al. (2009). As the Korotaevskiy et al. (2009) model considers both the classical and alternative pathways 
the latter was deactivated by making the plasma concentration of proteins involved in this pathway equal to zero. Secondly, the antigen-antibody complex was assumed to follow the kinetics shown in Eq. 14.

\subsubsection{Hole formation model}

Tschopp et al. (1984) found that the formation of one hole required $\alpha$ molecules of $\mathrm{C} 9, \alpha$ being in the range 12 to 18 . Hence, the number of holes formed was set to:

$$
h=V_{e} M \frac{N_{A}}{\alpha}
$$

where $V_{e}$ is the volume of the ECS, $M$ is the concentration of MAC and $N_{A}$ is the Avogadro number.

When holes are formed in the astrocytic membrane, ions are able to move freely across. Ionic currents through the lytic holes $\left(i_{h}\right)$ were assumed to be proportional to the Nernst potential as for models of standard ion channels by Endresen et al. (2000), as:

$$
i_{h}=h k_{h, P} \sinh \left(\frac{v_{c e l l}-E_{P}}{v_{t}}\right)
$$

where, $k_{h, i}$ is the permeability of one hole for ion $P v_{t}=\frac{R T}{F}, R$ is the gas constant, $T$ is temperature and $F$ is the Faraday constant. $k_{C}$ is defined as being proportional to the concentration of the $P$ channel with scaling factor of the ratio of the area of the hole to that of the channel leading to:

$$
k_{h, P}=\frac{k_{P}}{n_{P}}\left(\frac{r_{h}}{r_{P}}\right)^{2},
$$

where $k_{P}$ is the permeability of all ion channels of type $P, r_{h}$ is the radius of the hole, $r_{P}$ is the radius of the channel of type $P$ and $n_{P}$ is the number of channels of type $P$ in the membrane.

Similarly, holes are also made permeable to water. As holes are small, water flowing through the hole cannot be assumed to be a continuum. Hence, as in Eq. 18 the permeability has been assumed to be proportional to an AQP4 channel permeability with a constant equal to the ratio of the area of the hole to that of the channel $\left(r_{A Q P 4}\right)$, leading to:

$$
k_{h, w}=\frac{k_{A Q P 4}}{n_{A Q P 4}}\left(\frac{r_{h}}{r_{A Q P 4}}\right)^{2} .
$$

The flow of the water through holes is defined as:

$$
\begin{gathered}
Q_{h, c g}=h k_{h, w} \gamma\left[\Delta H_{c g}-\Delta \pi_{c g}\right], \\
Q_{h, g e}=h k_{h, w}(1-\gamma)\left[\Delta H_{g e}-\Delta \pi_{g e}\right],
\end{gathered}
$$


where, $Q_{h, c g}$ and $Q_{h, g e}$ is the water flow through the AQP4 perivascular and ECS interfaces respectively. The equations and parameters concerning channels in the model can be found in the supplementary material.

\subsubsection{CD59 kinetics}

CD59 is a complement inhibitor that is naturally found on the surfaces of cells including astrocytes. Many pathologies are associated with a lack of expression of this protein e.g. hemolytic anaemia (Brodsky, 2008) and breast cancer (Yu et al., 1999). Additionally, it is hypothesized by Saadoun and Papadopoulos (2015) that a down regulation of this protein at the astrocytic endfeet processes is the reason why these are targeted during NMO. The CD59 inhibits MAC formation by binding to C5b-8 and to C5b9, stopping further C 9 from attaching to the complex as presented in Figure 4.

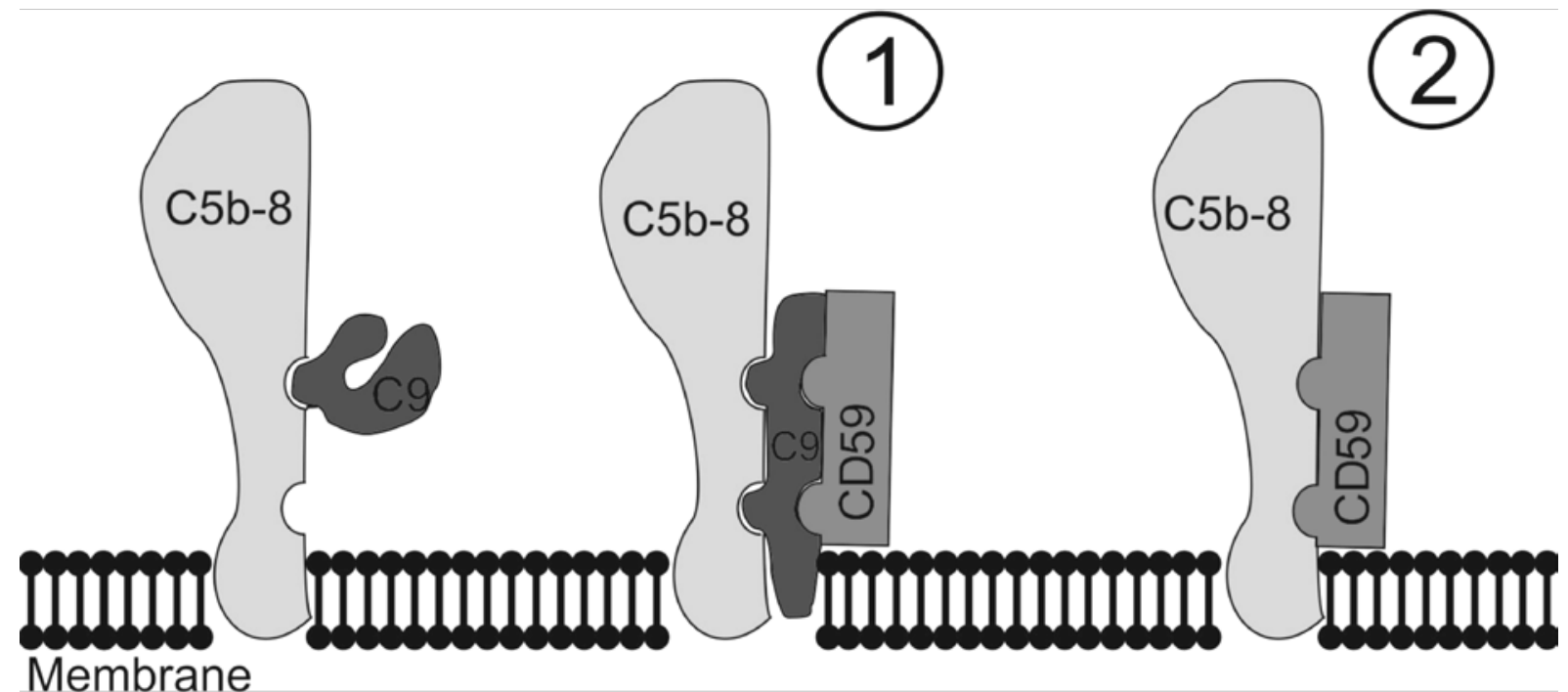

Figure 4: The protein CD59 inhibits the formation of MAC by blocking the binding of the perforating C9 proteins. CD59 is able to prevent the formation or during hole formation by binding to C5b-9 complex (1). Additionally, it can bind to C5b-8 and inhibits MAC formation (2) (Rollins et al., 1991).

The reduction of the rate of membrane lysis is assumed to be proportional to the reduction of MAC concentration. Furthermore, the bindings between CD59 and C5b678 and C5b6789 were defined using mass action kinetics as:

$$
\begin{gathered}
\stackrel{k_{1}}{\leftarrow} C_{b 5678+C D 59}^{\stackrel{\leftarrow}{\rightarrow}} C_{b 5678, C D 59} \\
k_{2} \\
k_{3} \\
M A C+C D 59 \underset{\rightarrow}{\leftarrow} C_{M A C, C D 59}, \\
k_{4}
\end{gathered}
$$

where $k_{1}, k_{2}, k_{3}$ and $k_{4}$ are the forward and backward reaction rates. Rollins et al., (1991) incorporated different concentrations of human CD59 into chicken E and then injected complement proteins derived from serum from different species (sheep, guinea 
pig, baboon, human). In particular, the serum contained proteins C5b6, C7, C8 and C9. To quantify the level of CD59 inhibition the levels of cell lysis were measured using radiolabelling.

To fit values for these parameters the data by Rollins et al., (1991). It consisted in measurements of the flow of stained particles through the membrane of chicken E cells membranes undergoing complement lysis in the presence of increasing concentrations of CD59.m It was assumed that the level of inhibition of flow was proportional to the concentration of MAC. As previously, the simplex method (Schmidt and Jirstrand, 2006) was used for the fit. The result of the fit is shown in Figure 5. Details of the fitting processes and values of parameters are given in the supplementary material.

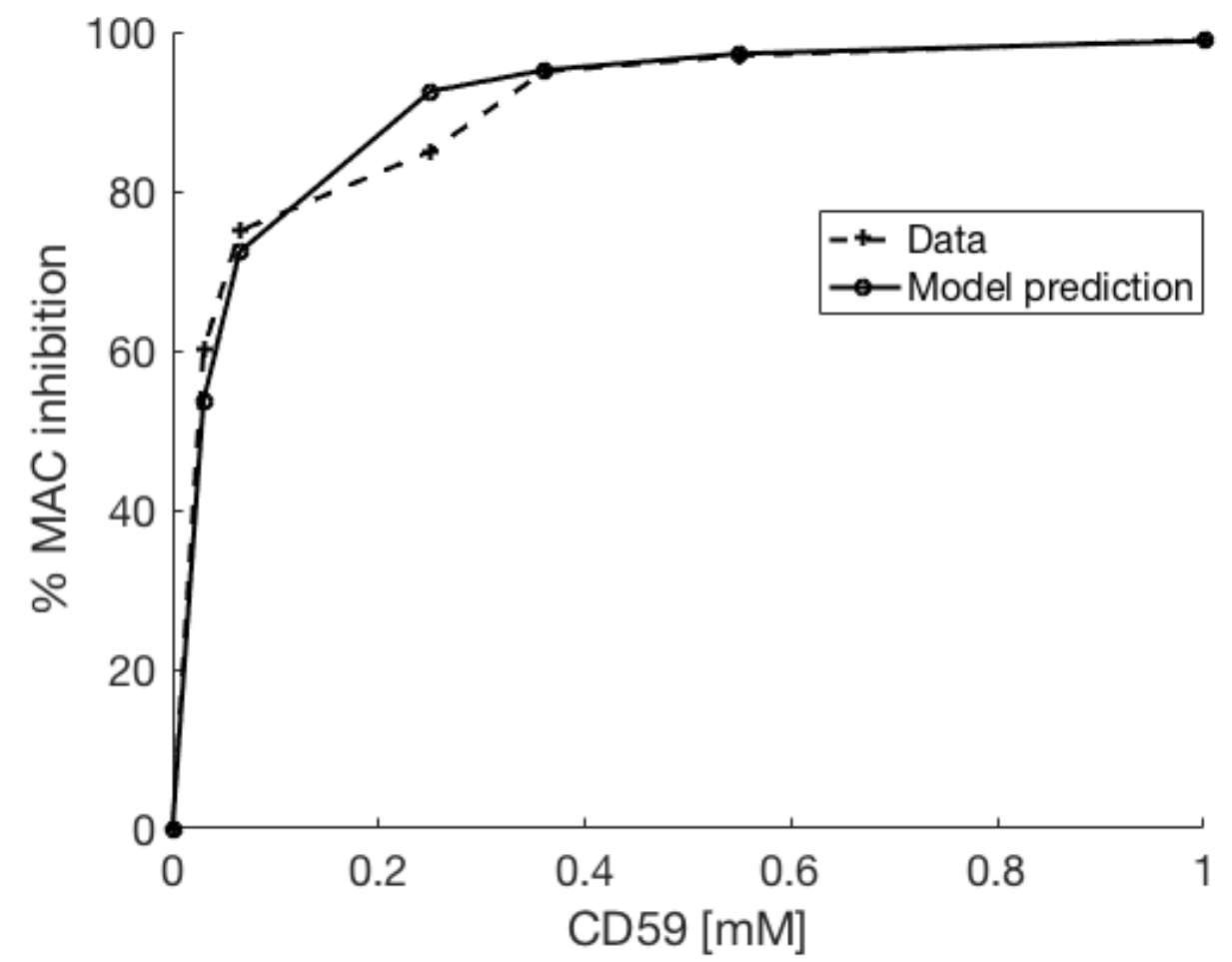

Figure 5: The result of the fitting of the complement lysis model augmented with CD59 kinetics to data in Rollins et al. (1991) showing the level of inhibition of MAC production by CD59.

\subsection{Sensitivity analysis}

As described in section 2.1 the Cloutier et al. model had to be refitted in order to be suitable for the simulations here performed. To assess the risk of over fitting, a screening algorithm was performed on the fitted model.

Due to its large size and the number of parameters it was decided to use a global rather than local method. As it is anticipated that this complex model will not be stable over the whole parameter space the global Campolongo-Morris (C-M) method was chosen.

This algorithm consists in exploring the parameter space by varying each parameter in turn over a pre-defined range (Salteli et al., 2004). Each time a parameter is varied its impact on the output of the model is evaluated, which is termed elementary effect (EE). The analysis is run over a series of EE calculations. From all the EEs standard deviation $(\sigma)$ and absolute mean $\left(\mu^{*}\right)$ are calculated for each parameter. The advantage of computing the absolute 
mean is that it captures the cumulative effect of varying each parameter in contrast to the mean, which would be affected by the signs of EEs. A large value of $\mu^{*}$ establishes that the parameter has a large effect on the output while those with a small value are unimportant. Additionally, the value of $\sigma$ can be used to distinguish between those parameters that have a linear independent effect (small $\sigma$ ) and those with a non-linear effect (large $\sigma$ ).

\subsection{Model implementation}

The cellular model was developed using the OpenCell platform (University of Auckland, New Zealand, v. 0.7). From the solvers available in the platform the Backwards Differential Formulas method with a maximum step size of 0.1 second was used.

In order to optimize the concentration of CD59 to inhibit complement lysis the model was also implemented in Matlab (version R2016a, Mathworks, MA, USA). To simulate the model on Matlab the 'ode15s' was used with absolute and relative error definitions set to $1 \times 10^{-6}$ and a maximum step size of 0.1 second. For simulations where the choice of parameters made the model too stiff for the 'ode15s' solver to converge the Backwards Differential Formulas method from CVODE package (Sundials, Lawrence Livermore National Institute, V.2.8.0) was used instead.

Furthermore, the simplex algorithm used for performing fits of parameters to data was implemented using the IQM SBML package (version V.2.1, Basel, Switzerland) developed by Schmidt and Jirstan (2006). Finally, sensitivity analysis of the results to changes in parameters was performed using the Campolongo-Morris method as described in Saltelli et al (2004).

\section{Results}

Two initial simulations were performed to show that the model makes reasonable predictions. First, it was shown that the model can predict cell swelling during stroke and; second, that it correctly identifies, as shown by experimental studies by Saadoun et al. (2010), that impairment of AQP4 channels is not the pathophysiological mechanism behind NMO, i.e. that their impairment does not cause swelling. Two subsequent simulations were then used to show the evolution of astrocyte swelling following complement and AQP4-Ab injection; and the potential of the inhibitory effect of CD59 on this process.

\subsection{Validating swelling model with stroke data}

Lundbaek and Hansen (1992) and Syková et al. (1994) in their experimental studies have shown that the ECS volume fraction falls to between 0.1-0.04 30 min after middle cerebral artery occlusion in a rat model that leads to complete reduction of blood flow through the brain. To simulate this level of ischemia blood flow through the capillary compartment in the model was reduced by $95 \%$.

The simulation showed that $30 \mathrm{~min}$ after stroke is induced the volume fraction of the ECS falls to approximately 0.046 , i.e. between the range reported in experimental data. The result of the simulation is shown in Figure 6. 


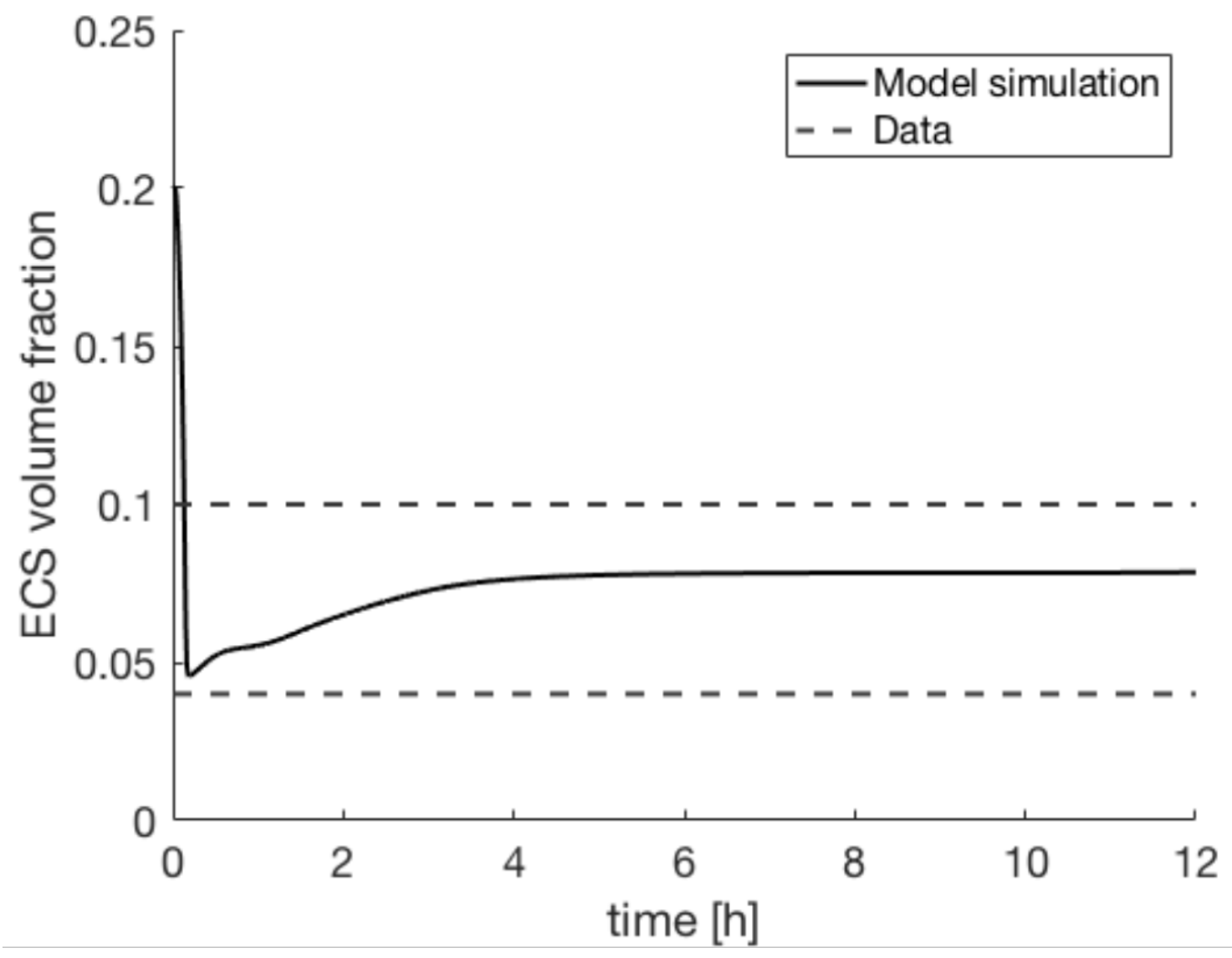

Figure 6: Simulation of the combined water dynamics model in Section 2.1 and metabolism and cell swelling model in Section 2.2. Red dotted lines show the range of ECS swelling, measured by Lundbaek and Hansen (1992) and Syková et al. (1994).

\subsection{AQP4 impairment}

Saadoun et al. (2010) tested experimentally whether astrocyte swelling is the result of AQP4 impairment due to the binding of AQP4-Ab. They found that if only AQP4-Ab is injected no significant swelling of astrocytes can be observed 12 hours after injection. To validate our model, we simulated how the cell volume is predicted to change after the deactivation of transport through AQP4 channels after 12 hours. The perivascular AQP4 and the AQP4 channels oriented towards the ECS were deactivated separately and together. The maximum volume change was of $1.2 \%$ when perivascular channels were deactivated.

\subsection{Complement lysis simulation}

Saadoun et al. (2010) found that only when both AQP4-Ab (16.8 $\mu$ l solution) and human complement (11.2 $\mu$ l solution) are injected NMO lesions develop. To simulate this experiment AQP4-Ab and human complement were set as input to the model. Their respective initial concentrations at the injection site were calculated based on solution preparation details in Saadoun et al. (2010) and the molar masses of AQP4-Ab (150 kDa) and human complement reported by $\mathrm{Li}$ et al., (2000) and Korotaevskiy et al. (2009) respectively. From these assumptions the initial concentration of AQP4-Ab was calculated to be $8.82 \times 10^{-5} \mathrm{mM} / \mathrm{L}$. Calculated concentrations of protein cascade proteins can be found in supplementary material. 
Model simulation span 12 hours to mimic the experimental work by Saadoun et al. (2010) who analysed histological data of the brain of mice models sacrificed after this time from injection. As shown in Figure 7, the model predicts that when no CD59 is injected after approximately one and half hour the volume reaches a steady state at $20 \%$ above its initial value. A maximum increase in the volume of $26 \%$ occurs after about 50 min.

\subsection{CD59 therapeutic potential}

The potential of CD59 as an astrocyte swelling inhibitor was tested by varying the ECS CD59 concentration and simulating the astrocyte volume change. Increasing the initial CD59 concentration resulted in a progressive increase of time after which the cell volume change plateau is reached (from $30 \mathrm{~min}$ to 3 hours for an increase in CD59 concentration from 0 to $1.5 \mathrm{mM}$ ) and a reduction of its value (from a $20 \%$ to a $17 \%$ volume increase plateau for an increase in CD59 concentration from 0 to $1.5 \mathrm{mM}$ ). Furthermore, the peak of the volume change is reduced (from an approximately $25 \%$ to a 20\% peak increase in astrocytic volume for a CD59 concentration of $0 \mathrm{mM}$ and $1.5 \mathrm{mM}$ respectively) as illustrated in Figure 7. The cell volume does not change within 12 hours of complement cascade onset for CD59 concentrations above $2.5 \mathrm{mM}$. This threshold is marked by the number of holes formed by MAC. Below approximately 40 holes the cell is able to stabilize and prevent membrane depolarization.

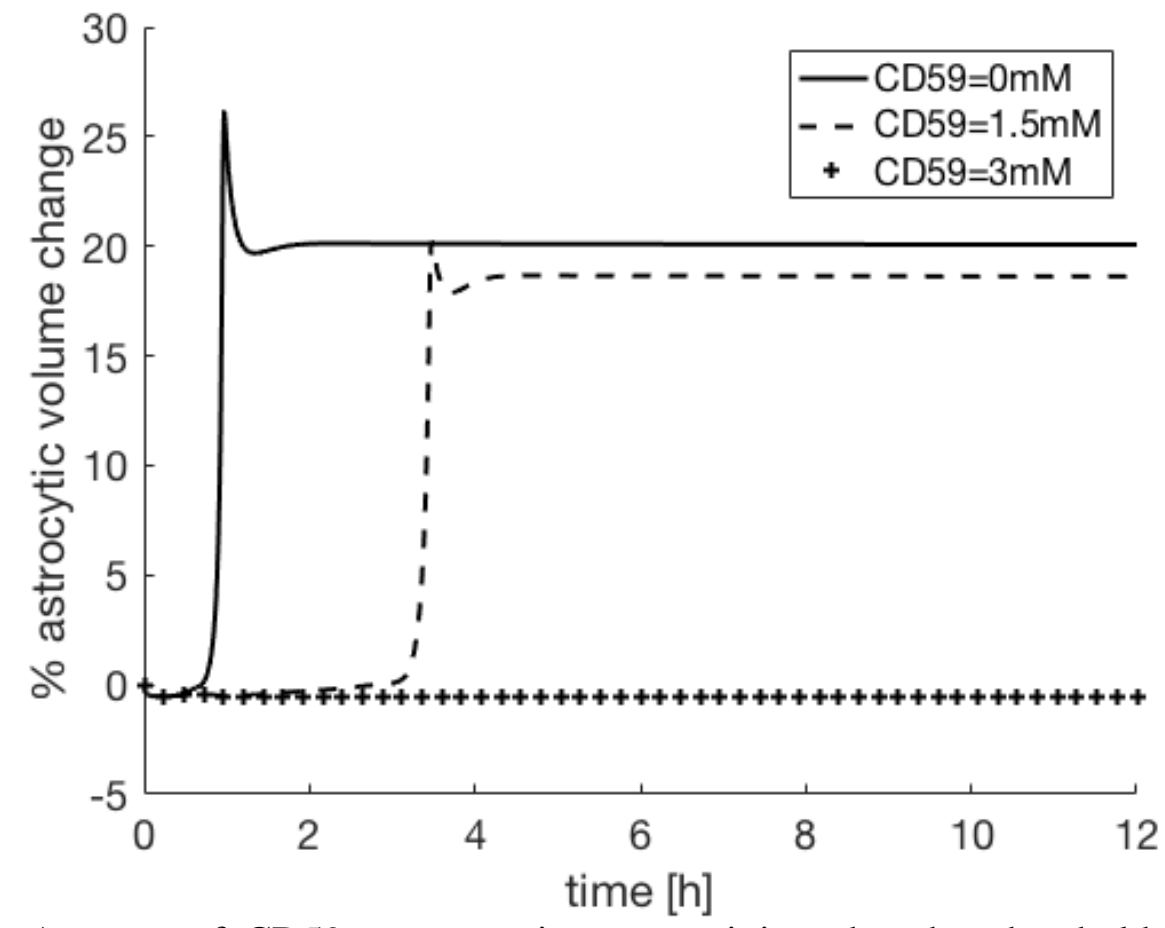

Figure 7: A range of CD59 concentrations were injected and a threshold for astrocytic swelling was found: below $2.5 \mathrm{mM}$ the membrane astrocytic membrane depolarizes inevitably leading to $20 \%$ volume increase while above $2.5 \mathrm{mM}$ astrocyte cytotoxic oedema is completely inhibited resulting in a negligible $0.1 \%$ volume increase.

\subsection{Screening results}

The method described in section 2.4 was applied to evaluate the sensitivity of the model predictions to the fitted parameters. It was applied to the validation of the 
cytotoxic oedema predictions with ischaemic stroke data by varying parameters by $\pm 5 \%$. The in vivo experimental works used for validation (Lundbaek and Hansen ,1992 and Syková et al., 1994) found that the ECS volume fraction is between 0.04 and 0.1 after 30 of ischamic stroke. For each iteration of the C-M algorithm stroke was simulated by reducing $\mathrm{CBF}$ by $95 \%$. In this case the $\mathrm{EE}$ was assumed to be the difference between the volume fraction of ECS after 30 min of stroke and the prediction with fitted parameters, as seen in Figure 6. From this analysis it was found that the maximum change was of \pm 2.5 percentage points resulting in the ECS volume fraction being between $4.49 \times 10^{-2}$ and $4.72 \times 10^{-2}$.

Additionally, the method was applied to the predicted CD59 complement lysis inhibition potential identified, as seen in Figure 7. The parameters fitted when developing the metabolic model (Section 2.2) as well as the parameters fitted to create the CD59 inhibition model (Section 2.3) were varied by $\pm 5 \%$. For each iteration it was evaluated if $2.5 \mathrm{mM}$ of CD59 prevented lysis within 12 hours of triggering the inflammatory complement cascade. The EE, at each iteration, is the difference between the volume fraction of astrocyte 12 hours after complement lysis is induced and the prediction presented in Figure 7. This analysis performed is summarised in Figure 8.

By analysing the results of the sensitivity analysis it was possible to divide the parameters into two categories: those that with a change of $5 \%$ result in the depolarisation of the astrocytic membrane even with the injection of $2.5 \mathrm{mM}$ of CD59 (Figure $8 \mathrm{I}$ ) and those that have a negligible impact on the threshold for CD59 inhibition of complement lysis (Figure 8 II).
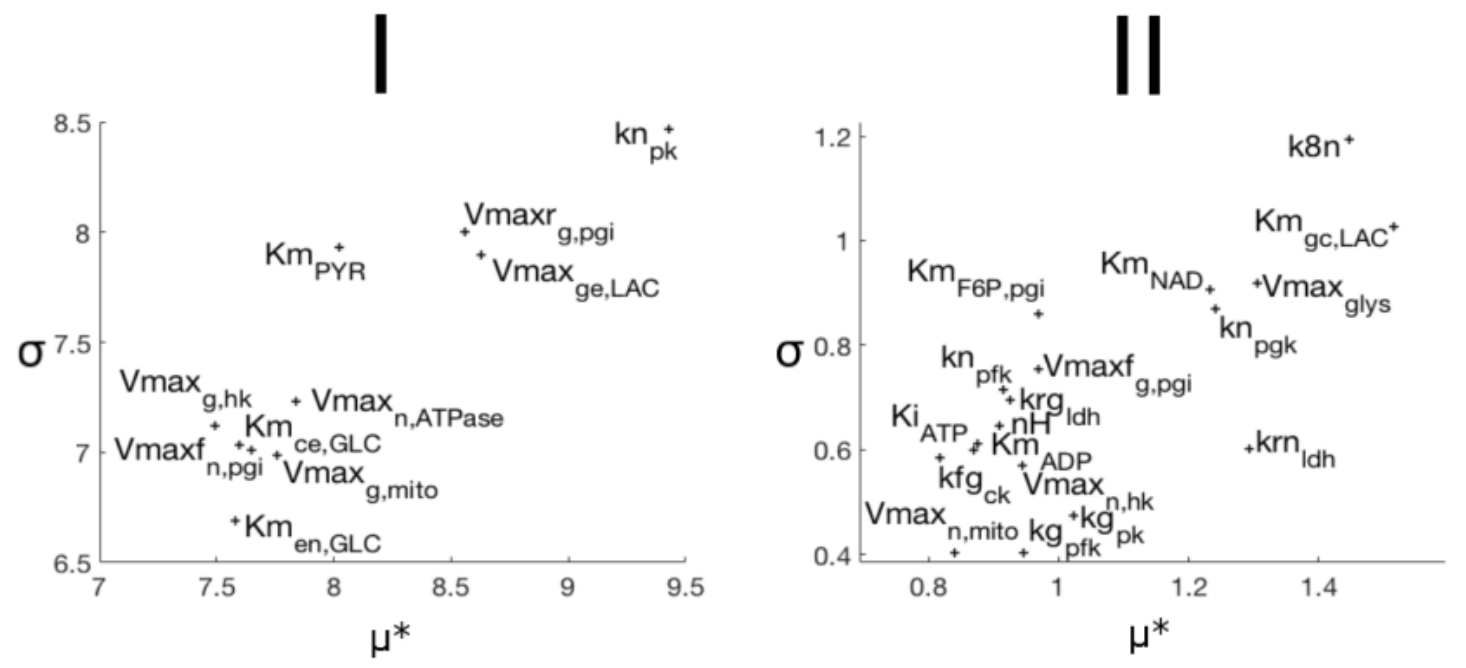

Figure 8: Outcome of applying the C-M sensitive analysis method to CD59 inhibitory predictions to the model where parameters are varied by $5 \%$. At each iteration of the C$\mathrm{M}$ method complement lysis was simulated for 12 hours when $2.5 \mathrm{mM}$ of CD59 are injected. The EE for was chosen to be the difference between the astrocytic volume fraction at 12 hours for each iteration parameter choice and the result presented in Figure 8. Two groups were found. I and II present the parameters that severely and negligibly impact the model predictions respectively.

\section{Discussion}


This work developed the first model of astrocyte swelling during NMO. The swelling is initiated by the injection of the NMO antibody and human complement into the ECS with respective concentrations replicating an experiment on a mouse model. The simulation predicts that the swelling occurs in one hour and leads to a volume increase of $25 \%$. The concentration of the injected human complement is $60 \%$ (Morgan and Harris, 1999) of that in the human plasma. Hence, the swelling process can be potentially faster depending on the mechanism of complement transport into the ECS during the condition. To appreciate the sensitivity of the evolution of the cell volume to a different initial complement concentration and to estimate the maximum rate of change of the cell volume during NMO, the model, with no CD59 added, was re-simulated with an ECS complement concentration equal to that of the plasma. i.e. a tentative estimate of the upper most value that a cell can experience during an episode of NMO. The complement concentration change resulted in the plateau volume being reached 5 minutes earlier compared to the $60 \%$ dilution simulation. This result suggests that dynamic change of cell volume during NMO will not take less than 1 hour. Hence, any experiment planning to characterize these dynamics would have to be designed with a matching temporal resolution.

Similarly increasing the concentration of AQP4-Ab up to three orders of magnitude above the initial value did not lead to significant changes of the rate of swelling. This is result is in agreement with the work of Liu et al. (2011) which shows that the activation of the classical complement pathway is not directly proportional to the antigen-antibody complex concentration.

From simulating complement cytotoxic oedema, it was found that the astrocyte volume experiences an exponential swelling as seen in Fig. 9. This behaviour is in line with the exponential production of MAC occurring in the complement cascade, as explained in Korotaevskiy et al. (2009). This behaviour is further confirmed by the experimental microscopy work by Schröder et al. (1990), who have shown that cellular conductance increases in steps of exponentially increasing magnitude every time a MAC hole is formed. Additionally, Schröder et al. (1990) fitted a model to the conductance curve measured where the membrane conductance $(i)$ is defined as:

$$
i(n)=i_{0}\left(N_{0}+N\right)^{2}
$$

where $i_{0}$ is the initial conductance, $N_{0}=1$ and $N$ is the number of holes formed. However, this analysis did not take into account the changes of the membrane permeability to each ion as was done in the model developed here.

The slow change of volume at the beginning of the simulation can be attributed to the sodium potassium pump managing to counterbalance the additional ionic flux occurring through membrane holes. This hyperactivity leads to a rapid depletion of ATP reserves and an inability for the pump to restore ionic balance with increasing currents due to more holes being created. As complement proteins are consumed the number of holes reaches a maximum of approximately 350 holes. This stabilizes the permeability of the membrane. Hence, after the exponential swelling the astrocyte volume reaches a peak of $25 \%$ increase. However, the peak is then followed by a decrease of $5 \%$ and the volume of the astrocyte stabilizes. This behaviour can be explained by the swelling of the neuron responding to changes in the osmotic pressure of the ECS. The evolution of the volume fraction occupied by each cell compartment is shown in Figure 9. 


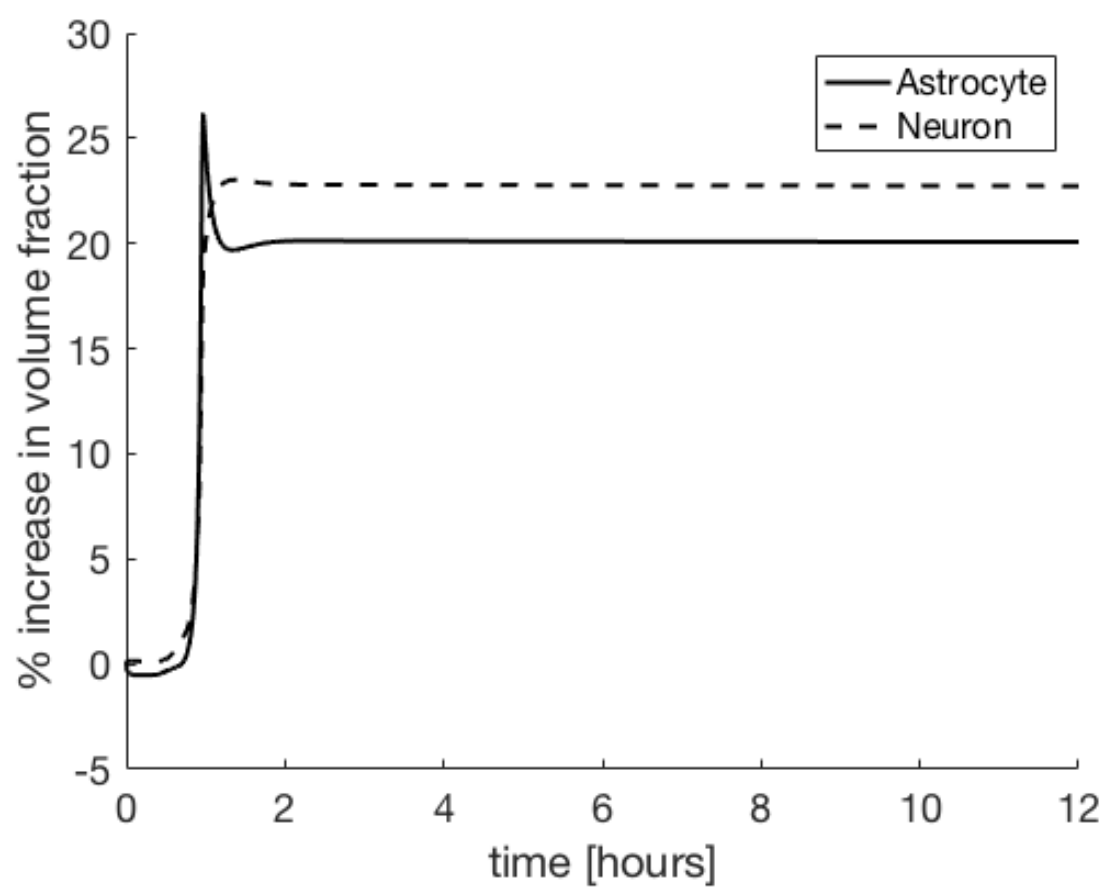

Figure 9: Comparison between the behaviour of astrocytic and neuron volume in the presence of astrocytic complement lysis.

The simulation of the injection of the CD59 MAC production inhibitor with AQP4$\mathrm{Ab}$ and human complement shows that there is a CD59 concentration threshold of 2.5 $\mathrm{mM}$ beyond which swelling can be completely prevented. This level of inhibition allows for only 40 holes to be created. As in this case the permeability of the cell is about 10 times lower than in the case where no CD59 is injected the sodium potassium pump is able to maintain ionic balance for at least 12 hours and no volume increase is observed.

When the initial CD59 concentration is below the $2.5 \mathrm{mM}$ threshold it inhibits the number of holes formed in the membrane compared to the no CD59 case. However, it cannot stop the sodium-potassium pump failure. The astrocyte volume peak is reduced as less holes means that the added membrane permeability is lower than in the no CD59 case. For the same reason the steady cell volume is also lower. These differences can be observed in Figure 9. This result was stress tested by applying the C-M method to this prediction where the parameters of the model are varied by $\pm 5 \%$.

Sensitivity analysis showed that the CD59 concentration threshold for complement lysis inhibition is sensitive to certain parameters such as $k n_{p k}$ andV $\max _{g e, L A C}$ from the metabolic model have determinant impact on the predictions of the model. These parameters define the production of ATP by the cell. Therefore, slight changes might result in the inability of the sodium potassium pump to maintain osmotic equilibrium when there is a slight increase in astrocytic membrane permeability due to lytic holes. Consequently, the membrane of the astrocyte depolarises and cell swells. The parameters fitted from Section 2.3 led to a maximum $\mu^{*}$ of $\pm 1 \%$. Therefore, measuring the parameters and kinetics of cellular metabolism is crucial to evaluate oedema in this and other pathological contexts. The importance of these parameters is further confirmed by the sensitivity analysis performed on the adapted metabolic model presented in the supplementary material.

Two further steps were taken in view of validating the model. Firstly, simulation of the combined model of metabolism and cell swelling in sections 2.1 and 2.2 in 
response to severe blood flow reduction in the capillary compartment was found to be in good agreement with the literature reporting that ECS volume fraction after $30 \mathrm{~min}$ of full ischemia goes down to between 0.04 and 0.1 . This result was stress tested by again applying the $\mathrm{C}-\mathrm{M}$ method. The parameters fitted for the developed metabolic model (Section 2.3) were varied by $\pm 5 \%$. It was found that for all simulations the ECS volume fraction after $30 \mathrm{~min}$ of full ischemia was between the boundaries experimentally measured. Therefore, ensuring confidence in the model predictions.

It would be desirable to further conduct a bifurcation analysis (BA) to characterise the transition in CD59 concentration leading to its therapeutic efficacy. However, as explained by Calderhead and Girolami (2011) BA becomes computationally expensive when applied to large and complex models and require prior knowledge of the impact of the model parameters on a studied output (Sumner et al., 2012). Both these constraints were prohibitive in our case.

The dynamic change of volume in both stroke and NMO simulations is rapid and occurs within a few hours. To improve validation, the model should be compared to data with multiple samples taken during that timeframe. Unfortunately, available data with such resolution are limited.

To improve validation a study could simultaneously track metabolites and cellular volume during ischemia and NMO. Examples of existing tools for these measurements include the use of CEST as in the work of Kogan et al. (2013) or ion selective electrodes as in the work of Syková et al. (1994).

Such new data could challenge some of the assumptions of the current model. For example, it may lead to changes being required in its metabolic section. A new model capturing neuronal metabolic function in the presence of Parkinson's disease by Poliquin et al., (2013) found that implementing kinetics of the full Krebs cycle was indispensable in order to achieve a satisfactory fit to data. A similar refinement may be needed here.

The complement cascade section of the model has its kinetics defined based on data gathered from exposure of bacteria to human complement (Korotaevskiy et al., 2009). The astrocyte is a bigger cell for which kinetics might be different. A dedicated experiment following the procedure described in the work of Korotaevskiy et al. (2009) should be performed on astrocytes to make the complement cascade model more representative of the NMO scenario.

Finally, the permeability of holes to water and ions has been defined in the model to be proportional to the permeability of AQP4 channels and that of ion channels respectively with a scaling factor defined as the ratio of the cross section area of the hole to that of the respective channel. An experiment is needed to define these permeabilities. Green and Goldberg, (1960) presented experimental work on complement effects on mammalian erythrocytes. It reported that within 5 minutes after complement activation with a large concentration of complement, $90 \%$ of erythrocyte intracellular potassium egressed while extracellular sodium and water entered the cytoplasm. It is here proposed that a similar experimental procedure should be implemented in order to measure ionic fluxes during astrocytic complement lysis.

In summary, kinetics of astrocyte swelling during NMO with the current model showed good qualitative agreement with existing data measuring water transport during complement lysis. The model was also in agreement with an experimental study showing that AQP4 impairment is not the primary cause of swelling during NMO. Furthermore, the metabolic and water transport sections of the model showed robustness to tests with available data characterizing swelling during ischemia. 
The introduced swelling model can be extend to study further important mechanisms. As shown in the work by Yang and Jiang (2017) and (Trappmann et al., 2012) changes in cell volume can lead to impairment of cell adhesion, which is important for wound healing, cell recognition and rigidity sensing. Additionally, as described by Stroka et al. (2014) AQP4 channels play an important role in astrocytic migration, which has been shown by Correale and Farez (2015) to play an important role in blood brain barrier permeability.

The model was used to test the inhibitory effect of CD59 on NMO induced astrocytic oedema. Simulations predicted that, to be effective, CD59 needs to be injected with a concentration beyond a threshold. Thus, the model shows potential for use in further predictive in silico studies applied to NMO. The complement cascade and complement lysis are pathological mechanisms generic to many brain conditions including ischaemia (Arumugam et al., 2004); neurodegenerative diseases such as Alzheimer's (Shen et al., 2013) and multiple sclerosis (Ingram et al., 2009)._Hence, this fundamental model can be repurposed for similar in silco studies in other contexts.

\section{References}

Arumugam, T.V., Shiels, I.A., Woodruff, T.M., Granger, D.N., Taylor, S.M., 2004. The role of the complement system in ischemia-reperfusion injury. Shock Augusta Ga 21, 401-409.

Blaivas, J.G., 1998. Conquering bladder and prostate problems: The authoritative guide for men and women. Plenum Press, Cambridge, Massachusetts, USA.

Brodsky, R.A., 2008. Narrative review: paroxysmal nocturnal hemoglobinuria: the physiology of complement-related hemolytic anemia. Ann. Intern. Med. 148, 587595.

Calderhead, B., Girolami, M., 2011. Statistical analysis of nonlinear dynamical systems using differential geometric sampling methods. Interface Focus rsfs 20110051. doi:10.1098/rsfs.2011.0051

Cloutier, M., Bolger, F.B., Lowry, J.P., Wellstead, P., 2009. An integrative dynamic model of brain energy metabolism using in vivo neurochemical measurements. J. Comput. Neurosci. 27, 391-414.

Correale, J., Farez, M.F., 2015. The Role of Astrocytes in Multiple Sclerosis Progression. Front. Neurol. 6.

Curtis, H., Barnes, N., 1989. Biology. Worth Publishers, New York, USA.

Davies, A., Lachmann, P.J., 1993. Membrane defence against complement lysis: the structure and biological properties of CD59. Immunol. Res. 12, 258-275.

Desai, B., Hsu, Y., Schneller, B., Hobbs, J.G., Mehta, A.I., Linninger, A., 2016. Hydrocephalus: the role of cerebral aquaporin-4 channels and computational modeling considerations of cerebrospinal fluid. Neurosurg. Focus 41, E8. doi:10.3171/2016.7.FOCUS16191

Dronne, M.-A., Boissel, J.-P., Grenier, E., 2006. A mathematical model of ion movements in grey matter during a stroke. J. Theor. Biol. 240, 599-615. 
Endresen, L., Hall, K., Høye, J., Myrheim, J., 2000. A theory for the membrane potential of living cells. Eur. Biophys. J. 29, 90-103.

Fujihara, K., Misu, T., Nakashima, I., Takahashi, T., Bradl, M., Lassmann, H., Takano, R., Nishiyama, S., Takai, Y., Suzuki, C., Sato, D., Kuroda, H., Nakamura, M., Fujimori, J., Narikawa, K., Sato, S., Itoyama, Y., Aoki, M., 2012. Neuromyelitis optica should be classified as an astrocytopathic disease rather than a demyelinating disease. Clin. Exp. Neuroimmunol. 3, 58-73. doi:10.1111/j.1759-1961.2012.00030.x

Green, H., Goldberg, B., 1960. The action of antibody and complement on mammalian cells. Ann. N. Y. Acad. Sci. 87, 352-362.

Hirayama, H., Yoshii, K., Ojima, H., Kawai, N., Gotoh, S., Fukuyama, Y., 1996. Linear systems analysis of activating processes of complement system as a defense mechanism. Biosystems 39, 173-185.

Ingram, G., Hakobyan, S., Robertson, N.P., Morgan, B.P., 2009. Complement in multiple sclerosis: its role in disease and potential as a biomarker. Clin. Exp. Immunol. 155, 128-139. doi:10.1111/j.1365-2249.2008.03830.x

Jiang, H., Sun, S.X., 2013. Cellular pressure and volume regulation and implications for cell mechanics. Biophys. J. 105, 609-619.

Jin, B.-J., Zhang, H., Binder, D.K., Verkman, A., 2013. Aquaporin-4-dependent K+ and water transport modeled in brain extracellular space following neuroexcitation. J. Gen. Physiol. 141, 119-132.

Kimelberg, H., 2004. Water homeostasis in the brain: basic concepts. Neuroscience 129, 851-860.

Kitley, J., Palace, J., 2016. Therapeutic options in neuromyelitis optica spectrum disorders. Expert Rev. Neurother. 16, 319-329. doi:10.1586/14737175.2016.1150178

Kleiter, I., Gahlen, A., Borisow, N., Fischer, K., Wernecke, K.-D., Wegner, B., Hellwig, K., Pache, F., Ruprecht, K., Havla, J., Krumbholz, M., Kümpfel, T., Aktas, O., Hartung, H.-P., Ringelstein, M., Geis, C., Kleinschnitz, C., Berthele, A., Hemmer, B., Angstwurm, K., Stellmann, J.-P., Schuster, S., Stangel, M., Lauda, F., Tumani, H., Mayer, C., Zeltner, L., Ziemann, U., Linker, R., Schwab, M., Marziniak, M., Then Bergh, F., Hofstadt-van Oy, U., Neuhaus, O., Winkelmann, A., Marouf, W., Faiss, J., Wildemann, B., Paul, F., Jarius, S., Trebst, C., Neuromyelitis Optica Study Group, 2016. Neuromyelitis optica: Evaluation of 871 attacks and 1,153 treatment courses. Ann. Neurol. 79, 206-216. doi:10.1002/ana.24554

Kogan, F., Hariharan, H., Reddy, R., 2013. Chemical exchange saturation transfer (CEST) imaging: description of technique and potential clinical applications. Curr. Radiol. Rep. 1, 102-114.

Korotaevskiy, A.A., Hanin, L.G., Khanin, M.A., 2009. Non-linear dynamics of the complement system activation. Math. Biosci. 222, 127-143.

Kumagai, I., Tsumoto, K., 2010. Antigen-antibody binding. Encycl. Life Sci. 1-7.

Kumar, V., Abbas, A.K., Aster, J.C., 2012. Robbins basic pathology. Elsevier Health Sciences, Philadelphia, USA.

Lewis, L.A., Ram, S., 2014. Meningococcal disease and the complement system. Virulence 5, 98-126. doi:10.4161/viru.26515

Li, L., Kalaga, R., Paul, S., 2000. Proteolytic components of serum IgG preparations. Clin. Exp. Immunol. 120, 261-266. doi:10.1046/j.1365-2249.2000.01219.x

Liu, B., Zhang, J., Tan, P.Y., Hsu, D., Blom, A.M., Leong, B., Sethi, S., Ho, B., Ding, J.L., Thiagarajan, P., 2011. A computational and experimental study of the regulatory mechanisms of the complement system. PLoS Comput Biol 7, e1001059. 
Lundbaek, J., Hansen, A., 1992. Brain interstitial volume fraction and tortuosity in anoxia. Evaluation of the ion-selective micro-electrode method. Acta Physiol. Scand. 146, 473-484.

Morgan, B.P., Harris, A.L., 1999. Complement Regulatory Proteins. Academic Press.

Nagelhus, E.A., Ottersen, O.P., 2013. Physiological roles of aquaporin-4 in brain. Physiol. Rev. 93, 1543-1562.

Orlowski, P., O’Neill, D., Grau, V., Ventikos, Y., Payne, S., 2013. Modelling of the physiological response of the brain to ischaemic stroke. Interface Focus 3, 20120079.

Østby, I., Øyehaug, L., Einevoll, G.T., Nagelhus, E.A., Plahte, E., Zeuthen, T., Lloyd, C.M., Ottersen, O.P., Omholt, S.W., 2009. Astrocytic mechanisms explaining neuralactivity-induced shrinkage of extraneuronal space. PLoS Comput Biol 5, e1000272.

Papadopoulos, M.C., Bennett, J.L., Verkman, A.S., 2014. Treatment of neuromyelitis optica: state-of-the-art and emerging therapies. Nat. Rev. Neurol. 10, 493-506. doi:10.1038/nrneurol.2014.141

Pittock, S.J., Lennon, V.A., McKeon, A., Mandrekar, J., Weinshenker, B.G., Lucchinetti, C.F., O’Toole, O., Wingerchuk, D.M., 2013. Eculizumab in AQP4-IgG-positive relapsing neuromyelitis optica spectrum disorders: an open-label pilot study. Lancet Neurol. 12, 554-562. doi:10.1016/S1474-4422(13)70076-0

Poliquin, P.O., Chen, J., Cloutier, M., Trudeau, L.-É., Jolicoeur, M., 2013. Metabolomics and in-silico analysis reveal critical energy deregulations in animal models of Parkinson's disease. PloS One 8, e69146.

Rollins, S.A., Zhao, J., Ninomiya, H., Sims, P.J., 1991. Inhibition of homologous complement by CD59 is mediated by a species-selective recognition conferred through binding to C8 within C5b-8 or C9 within C5b-9. J. Immunol. Baltim. Md 1950 146, 2345-2351.

Saadoun, S., Papadopoulos, M.C., 2015. Role of membrane complement regulators in neuromyelitis optica. Mult. Scler. J. 21, 1644-1654.

Saadoun, S., Waters, P., Bell, B.A., Vincent, A., Verkman, A., Papadopoulos, M.C., 2010. Intra-cerebral injection of neuromyelitis optica immunoglobulin $\mathrm{G}$ and human complement produces neuromyelitis optica lesions in mice. Brain 133, 349-361.

Schmidt, H., Jirstrand, M., 2006. Systems Biology Toolbox for MATLAB: a computational platform for research in systems biology. Bioinformatics 22, 514-515.

Schröder, G., Brandenburg, K., Brade, L., Seydel, U., 1990. Pore formation by complement in the outer membrane of gram-negative bacteria studied with asymmetric planar lipopolysaccharide/phospholipid bilayers. J. Membr. Biol. 118, 161-170.

Shen, Y., Yang, L., Li, R., 2013. What does complement do in Alzheimer's disease? Old molecules with new insights. Transl. Neurodegener. 2, 21. doi:10.1186/20479158-2-21

Solenov, E., Watanabe, H., Manley, G.T., Verkman, A., 2004. Sevenfold-reduced osmotic water permeability in primary astrocyte cultures from AQP-4-deficient mice, measured by a fluorescence quenching method. Am. J. Physiol.-Cell Physiol. 286, C426-C432.

Stroka, K.M., Jiang, H., Chen, S.-H., Tong, Z., Wirtz, D., Sun, S.X., Konstantopoulos, K., 2014. Water permeation drives tumor cell migration in confined microenvironments. Cell 157, 611-623. 
Sumner, T., Shephard, E., Bogle, I.D.L., 2012. A methodology for global-sensitivity analysis of time-dependent outputs in systems biology modelling. J. R. Soc. Interface 9, 2156-2166. doi:10.1098/rsif.2011.0891

Syková, E., Svoboda, J., Polák, J., Chvátal, A., 1994. Extracellular volume fraction and diffusion characteristics during progressive ischemia and terminal anoxia in the spinal cord of the rat. J. Cereb. Blood Flow Metab. 14, 301-311.

Trappmann, B., Gautrot, J.E., Connelly, J.T., Strange, D.G.T., Li, Y., Oyen, M.L., Cohen Stuart A., M., Boehm, H., Li, B., Vogel, V., Spatz, J.P., Watt, F.M., Huck, W.T.S., 2012.

Extracellular-matrix tethering regulates stem-cell fate. Nat. Mater. 11, 642-649.

Tschopp, J., Engel, A., Podack, E., 1984. Molecular weight of poly (C9). 12 to 18 C9 molecules form the transmembrane channel of complement. J. Biol. Chem. 259, 1922-1928.

Yang, Y., Jiang, H., 2017. Shape and Dynamics of Adhesive Cells: Mechanical Response of Open Systems. Phys. Rev. Lett. 118, 208102.

Yu, J., Caragine, T., Chen, S., Morgan, B., Frey, A., Tomlinson, S., 1999. Protection of human breast cancer cells from complement-mediated lysis by expression of heterologous CD59. Clin. Exp. Immunol. 115, 13.

Zipfel, P.F., Skerka, C., 2009. Complement regulators and inhibitory proteins. Nat. Rev. Immunol. 9, 729-740. 